\title{
Theory of Anisotropic Hopping Transport due to Spiral Correlations in the Spin-Glass Phase of Underdoped Cuprates
}

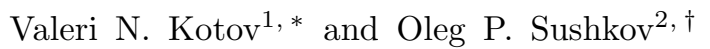 \\ ${ }^{1}$ Institute of Theoretical Physics, Swiss Federal Institute of Technology (EPFL), \\ CH-1015 Lausanne, Switzerland \\ ${ }^{2}$ School of Physics, University of New South Wales, Sydney 2052, Australia
}

\begin{abstract}
We study the in-plane resistivity anisotropy in the spin-glass phase of the high- $T_{c}$ cuprates, on the basis of holes moving in a spiral spin background. This picture follows from analysis of the extended $t-J$ model with Coulomb impurities. In the variable-range hopping regime the resistivity anisotropy is found to have a maximum value of around $90 \%$, and it decreases with temperature, in excellent agreement with experiments in $\mathrm{La}_{2-x} \mathrm{Sr}_{x} \mathrm{CuO}_{4}$. In our approach the transport anisotropy is due to the non-collinearity of the spiral spin state, rather than an intrinsic tendency of the charges to self-organize.
\end{abstract}

\section{INTRODUCTION}

One of the central issues in the physics of the high-temperature superconductors is the nature of the ground state at low doping. In particular, the possible co-existence of ordering tendencies in the spin and charge sectors at low temperature is currently being actively investigated 1.2 .3 Experimentally at low temperature $\mathrm{La}_{2-x} \mathrm{Sr}_{x} \mathrm{CuO}_{4}$ (LSCO) co-doped with Nd (LNSCO) exhibits static lattice deformation ${ }^{4}$ as well as incommensurate $(\mathrm{IC})$ magnetic order at doping $x \approx 1 / 8$. The lattice deformation indicates the presence of static charge order (stripes). Recently similar behavior has also been found in $\mathrm{La}_{2-x} \mathrm{Ba}_{x} \mathrm{CuO}_{4}$ (LBCO),$\frac{5}{5} x \approx 1 / 8$. However for $x<1 / 8$ and in Nd-free LSCO, charge order has not been detected, while IC magnetism persists down to $x \approx 0.02{ }^{6.7}$ Thus while IC magnetic order is generically observed in the underdoped regime, static charge order seems to be confined to the neighborhood of $x=1 / 8$.

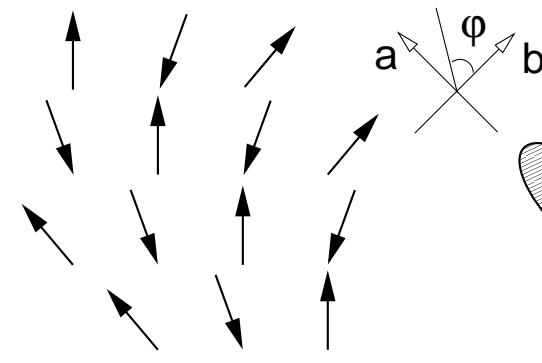

(a)

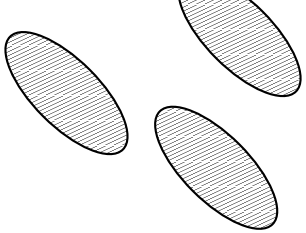

(b)
FIG. 1: (a) (1,1) Spiral spin state. (b) Localized (Coulomb trapped) holes, with positions pinned by the random $\mathrm{Sr}$ impurities (schematic). The ellipses reflect the symmetry of the wave-function (6). The orthorhombic $\hat{a}$ and $\hat{b}$ directions are shown.

Theoretically the IC magnetism in the cuprates is often modeled as originating from static charge stripes, and since these are not universally present, one is forced to introduce additional concepts, such as "fluctuating" stripes, electronic liquid crystals, etc . $^{3.8}$ The microscopic origin of such states is still not clear and is currently being debated. An alternative explanation for the IC magnetism which follows naturally from the $t-J$ model, is the formation of a non-collinear spiral state, partially relieving the frustration due to the hole motion. 9 Such a state would not co-exist with charge order, as can be shown in the context of effective Landau theory. ${ }^{10}$ However it is still possible that in the presence of anisotropic Dzyaloshinski-Moriya interactions the spin spiral could cause weak lattice modulation with period generically half that of the spiral. While the spiral ground state has a tendency to be unstable toward phase separation, we have shown recently that in the extended $t-t^{\prime}-t^{\prime \prime}-J$ model the spiral can be stabilized by the presence of the additional hoppings 11 The spiral description was successfully applied to explain magnetic properties of LSCO, such as the location of the elastic neutron scattering peaks and the change of the incommensurability direction by $45^{\circ}$ across the superconductor-insulator boundary $(x=0.055), 12$ The spiral was also proposed as a candidate for the ground state in the spin-glass (insulating) phase for $0.02<x<0.055^{12,13,14}$

In the present work we address transport properties within the spiral framework. Our main motivation comes from the recent experimental data in the spin-glass phase of LSCO showing transport anisotropies as large as 50\% to $70 \%$, both in DC and AC measurements ${ }^{15.16}$ It has been argued that these data provide indirect support to the notion of fluctuating stripes or electronic liquid crystals, but no quantitative theory exists that takes these concepts into account ${ }^{3}$ Recent infrared experiments that carefully identify phonon modes in LSCO once again find no charge ordering tendencies in the spin-glass phase of this material. 17 Therefore we take the point of view that the ground state has a spiral spin structure, and calculate the transport anisotropy in the variable-range hopping $(\mathrm{VRH})$ regime for low temperature and frequency, where the anisotropy has the largest value. We show that the spatial anisotropy of the hole wave-function in a spiral translates into anisotropy of the hopping transport. Our main result is that for microscopic parameters appropriate for LSCO (within the $t-t^{\prime}-t^{\prime \prime}-J$ model), the mag- 
nitude of the anisotropy is large (40\%-90\%, depending on temperature), and close to the one found experimentally. Thus we demonstrate that the transport anisotropy data can be explained quantitatively within the spiral theory which does not involve any tendency of the holes to selforganize into charge stripes. The anisotropy was also analyzed in Ref. 14 on the basis of topological defect scattering in a spiral; however the results are applicable to the quasi-metallic (higher temperature) regime where the anisotropy is very small, of the order of several percent. We emphasize that in the present work we consider the low-temperature, strongly-localized regime where it is clear from experiment that the anisotropy is the largest (10-20 times larger than the value in the metallic regime). Finally we mention that the problem was also addressed within the spin-charge separation scenario which provides a description of the pseudogap phase $\frac{18}{}$ and could lead to effective "insulating" behavior; however within this approach the transport anisotropy is linked to the magnetic correlation length anisotropy which can be taken from experiment but cannot be explicitly calculated.

\section{A. Summary of Previous Results and Notation}

Our starting point is the description of the spin-glass phase $(0.02<x<0.055)$ developed in Ref. 12 which is briefly summarized below. First, a single hole resides near the points $\mathbf{k}_{0}=( \pm \pi / 2, \pm \pi / 2)$, with a quadratic dispersion around them $\epsilon_{\mathbf{k}} \approx \frac{\beta_{1}}{2} k_{1}^{2}+\frac{\beta_{2}}{2} k_{2}^{2}$, where $k_{1}$ is perpendicular to the face of the magnetic Brillouin zone and $k_{2}$ is parallel to the face. Within the effective $t-t^{\prime}-t^{\prime \prime}-J$ model for LSCO, the parameters are taken to be: $t / J=$ $3.1, t^{\prime} / J \approx-0.5, t^{\prime \prime} / J \approx 0.3$, where $J \approx 125 \mathrm{meV}$. From now on we measure energies in units of $J$ (i.e. set $J=1$ ) and lengths in units of the lattice spacing $a$ (we set $a=1$ ). By using the self-consistent Born approximation one finds that these parameters lead to an almost isotropic dispersion $\beta_{1} \approx \beta_{2}=\beta \approx 2.2$, and a quasiparticle residue around the nodal points $Z \approx 0.34$. A hole trapped by the Coulomb field of the Sr ion generates a spiral distortion of the Néel background, which can be parametrized as: $|\mathbf{i}\rangle=e^{i \theta\left(\mathbf{r}_{\mathbf{i}}\right) \mathbf{m} \cdot \sigma / 2}|\uparrow\rangle,|\mathbf{j}\rangle=e^{i \theta\left(\mathbf{r}_{\mathbf{j}}\right) \mathbf{m} \cdot \sigma / 2}|\downarrow\rangle$, $\mathrm{i} \in$ "up" sublattice, $\mathrm{j} \in$ "down" sublattice, where $\mathbf{m}$ is an arbitrary unit vector perpendicular to the quantization axis of the states $|\uparrow\rangle,|\downarrow\rangle$. The angle $\theta(\mathbf{r})$, measuring deviations from collinearity is given by 12

$$
\theta(\mathbf{r})=\frac{Z t}{\sqrt{2} \pi \rho_{s}} \frac{\left(\mathbf{e}_{ \pm} \cdot \mathbf{r}\right)}{r^{2}}\left[1-e^{-2 \kappa r}(1+2 \kappa r)\right]
$$

where $\rho_{s} \approx 0.18$ is the spin stiffness, and $1 / \kappa \sim 3-5$ is the localization length of the orbital part of the wave function. The value of $1 / \kappa$ is extracted from experimental data $\frac{12}{12}$ and is generally expected to increase with doping. The unit vector $\mathbf{e}_{ \pm}=\frac{1}{\sqrt{2}}(1, \pm 1)$, in the usual square-lattice coordinate system. At finite doping $(0.02<x<0.055)$ the interaction of the long-range dipole distortions from holes trapped at different $\mathrm{Sr}$ ions leads to spiral magnetic order ${ }^{12}$ characterized by average $\bar{\theta} \propto \mathbf{e}_{ \pm} \cdot \mathbf{r}$, as shown in Fig. 11(a). In a perfect square lattice the spiral can be directed along any diagonal $(1, \pm 1)$. However from the location of the elastic neutron scattering peaks ${ }^{6}$ the incommensurability is determined to be along the orthorhombic $\hat{b}$ direction. In our picture this means that the orthorhombic deformation pins the direction of the spiral, as shown in Fig. 1 and thus we set $\mathbf{e}=\mathbf{e}_{+}=\frac{1}{\sqrt{2}}(1,1)$. In what follows the exact nature of the pinning mechanism, to be discussed elsewhere, will not be important.

Experimentally the magnetic correlation length is finite $\xi \sim 6-20$ (decreases with increasing doping) $\stackrel{6.7}{=}$ On the theoretical side it was argued that $\xi$ is finite due to topological defects that lead to frustration of the longrange spiral order ${ }^{12.13}$ Since the localization length is less than the magnetic scale, $1 / \kappa<\xi$, we expect that the effective description of transport properties, developed below in terms of the one-hole wave function, is quantitatively valid also at finite (small) doping (i.e. the topological frustration mechanism does not affect our considerations). It should be noted that the above inequality while being explicit is also the strongest (most restrictive) condition we could give. Its refinement would have to come out of a detailed theory of IC magnetism in the spin-glass phase (i.e. a self-consistent theory that takes into account both the effective disorder and the spiral formation, generated by the doped holes). Purely theoretical arguments aside, transport measurements in the doping range $0 \%-4 \%$ suggest that the system is in a strongly-localized regime at low temperatures, and we thus expect our calculations to be valid as long as doping is not too close to the insulator-metal boundary (at $5.5 \%)$.

The rest of the paper is organized as follows. In Section II we analyze the properties of a localized hole in a spiral background, and in Section III we use the hole's wave function to calculate the in-plane transport anisotropies in the variable-range hopping regime. Section IV contains our conclusions.

\section{LOCALIZED HOLE IN THE PRESENCE OF MAGNETIC SPIRAL CORRELATIONS}

The coupling $H_{S P}$ between the spin of the magnetic background (angle $\theta(\mathbf{r})$ ) and the orbital wave-function of the hole $\chi(\mathbf{r})$ generates the spiral and has the form: ${ }^{12}$

$$
H_{S P}=-\sqrt{2} Z t \int(\mathbf{e} \cdot \nabla \theta) \chi^{2}(\mathbf{r}) d^{2} r .
$$

The effective Schrödinger equation is then:

$$
\left(-\frac{\beta}{2} \nabla^{2}-\frac{q^{2}}{r}-\sqrt{2} Z t(\mathbf{e} \cdot \nabla \theta)\right) \chi(\mathbf{r})=\epsilon \chi(\mathbf{r}) .
$$

Here the Coulomb potential of the $S r$ ion which keeps the hole localized is $\frac{q^{2}}{r}=\frac{q_{0}^{2}}{\mathcal{E}_{e} r}$, where $q_{0}$ is the unit charge and 
$\mathcal{E}_{e}$ is the effective dielectric constant known to be quite large in the copper oxides, $\mathcal{E}_{e} \sim 30-100$ (increases with doping), 19 The last term in (3) determines the anisotropy

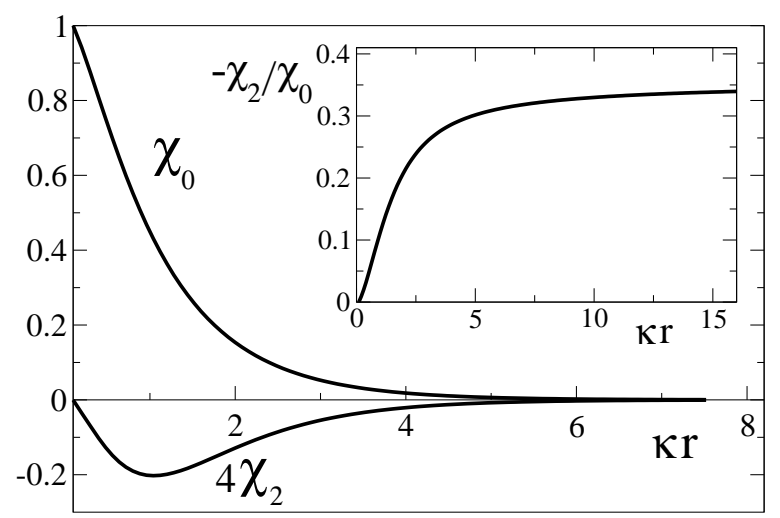

FIG. 2: Solution of (3) in terms of the expansion (6), for $\Lambda=$ 1. Only the first two (largest) components are shown. The normalization of $\chi_{0}$ is arbitrary. Inset: The ratio $-\chi_{2} / \chi_{0}$.

of $\chi(\mathbf{r})$ and is given by

$$
\begin{aligned}
& \sqrt{2} Z t(\mathbf{e} \cdot \nabla \theta)=\frac{Z^{2} t^{2}}{\pi \rho_{s}} \frac{1}{r^{2}}[f(\kappa r)-g(\kappa r) \cos (2 \varphi)], \\
& g(z)=1-\left(1+2 z+2 z^{2}\right) e^{-2 z}, f(z)=2 z^{2} e^{-2 z} .
\end{aligned}
$$

The coordinate system is chosen so that $\mathbf{e}$ is parallel to the $\hat{b}$ axis and $\varphi$ is the polar angle (Fig. 11). The value of $\kappa$ (and consequently $\epsilon$ ) can be found by a variational minimization of the total energy: ${ }^{12}$

$$
\kappa=\frac{2 q^{2} / \beta}{1-\frac{\Lambda}{2}}, \epsilon=-\beta \kappa^{2} / 2, \quad \Lambda \equiv \frac{Z^{2} t^{2}}{\pi \beta \rho_{s}} .
$$

We will use $\kappa \sim 0.3-0.4$, consistent with experiment, 12 rather than rely on (5) which contains the uncertainty related to the exact value of the dielectric constant. The dimensionless parameter $\Lambda$, as defined in (5), characterizes the coupling between the orbital motion of the hole and the deformation of the spin background. Substituting the values of $Z, \beta$ appropriate for LSCO (discussed in Section I.A), we obtain $\Lambda \approx 1$, which will be used from now on.

Let us look for solution of (3) in terms of the series in angular harmonics

$\chi(\kappa r, \varphi)=\chi_{0}(\kappa r)+\chi_{2}(\kappa r) \cos (2 \varphi)+\chi_{4}(\kappa r) \cos (4 \varphi)+\ldots$

Then Eq. (3) leads to a set of coupled equations for $\chi_{i}, i=0,2,4, \ldots$ We have solved these equations numerically and the results for the first two harmonics are presented in Fig. 2 The next harmonics are found to be small, for example $\chi_{4} / \chi_{0}<2 \times 10^{-2}$, and can be in fact safely neglected. At large distances all the wave-functions behave as $\chi_{i} \propto e^{-\kappa r}$, while at finite distances this behavior is substantially modified, as shown in Fig. 2]

\section{TRANSPORT ANISOTROPIES IN THE VARIABLE-RANGE HOPPING (VRH) REGIME}

\section{A. In-plane DC Resistivity Anisotropy}

Below characteristic temperature $T_{V R H} \approx 20-30 \mathrm{~K}$, the low-temperature resistivity of LSCO in the spin-glass phase can be described by the $2 \mathrm{D}$ version of the Mott VRH formula $\rho \sim \exp \left(T_{0} / T\right)^{1 / 3}, 19.20 .21 .22$ Here $T_{0}$ depends somewhat on doping and sample quality and generally decreases when doping increases (and thus conduction becomes easier). As an estimate, for example at $4 \%$ doping the data of Ref. 20 are well fit with $T_{0} \approx 500 \mathrm{~K} ; \stackrel{21}{ }$ analyzing the curves of Ref. 22 we obtain $T_{0} \approx 200-300 \mathrm{~K}$, and from Ref. 15 we have extracted $T_{0} \approx 200 \mathrm{~K}$.

Within the spiral framework the physical idea behind the resistivity anisotropy is quite simple. According to (6) the hole wave function acquires an elliptic deformation induced by the spin spiral, as shown schematically in Fig. 1(b). Hence hopping in the $\hat{b}$-direction is less probable than hopping in the $\hat{a}$-direction. Using (6), and essentially following the derivation of Mott's result, 23 we obtain for the resistivity anisotropy:

$$
\begin{aligned}
& \frac{\rho_{b}(T)}{\rho_{a}(T)}=\frac{\int_{0}^{2 \pi} \sin ^{2}(\varphi) \Phi^{2}\left(\kappa r_{T}, \varphi\right) d \varphi}{\int_{0}^{2 \pi} \cos ^{2}(\varphi) \Phi^{2}\left(\kappa r_{T}, \varphi\right) d \varphi} \\
& \Phi(\kappa r, \varphi) \equiv \frac{\chi(\kappa r, \varphi)}{\chi_{0}(\kappa r)} .
\end{aligned}
$$

Here $r_{T}=\frac{1}{2 \kappa}\left(T_{0} / T\right)^{1 / 3}$ is the VRH length, ${ }^{23}$ and in order for the approach to be justified we must certainly have $\kappa r_{T}>1$. Eq. (7) reflects the difference in the wavefunction overlap for the two electric field directions. The factors $\sin ^{2}(\varphi), \cos ^{2}(\varphi)$ arise from the fact that the conductivity varies with the square of the carrier jump distance projection in the electric field direction. ${ }^{23}$ Since the hole's dispersion is isotropic $\left(\beta_{1}=\beta_{2}=\beta\right)$, the exponential part of the wave function is isotropic and consequently the resistivity anisotropy is expected to arise from the anisotropy of the exponential prefactors, whose overlap leads to (7). Both the VRH length and $T_{0}$ thus remain isotropic within this formulation.

It is clear from (7) that as $T$ decreases (i.e. $\kappa r_{T}$ increases) the anisotropy grows, due to the increase of $\left|\chi_{2} / \chi_{0}\right|$ (Fig. 2(Inset)) and consequently the more pronounced angular dependence of $\Phi\left(\kappa r_{T}, \varphi\right)$. The results are summarized in Fig. 3. where also the evolution of the zero temperature anisotropy $\rho_{b}(T=0) / \rho_{a}(T=0)$ as a function of $\Lambda$ is shown for completeness in the inset. At very low temperature $\left(T \lesssim 1 \mathrm{~K}, \frac{21}{2}\right.$ i.e. $\left.T / T_{0} \lesssim 10^{-3}\right)$ we would have to take into account a crossover to the Coulomb gap regime, which however would practically not influence the curve in Fig. 3

The data of Ref. 15 were taken at temperatures $T>$ $10 \mathrm{~K}$, meaning that the lowest ratio $T / T_{0} \sim 0.05$ (we take $\left.T_{0} \sim 200 \mathrm{~K}\right)$. As the temperature increases beyond $T_{V R H} \approx 20-30 \mathrm{~K}$ when $\kappa r_{T} \approx 1$, the approach 


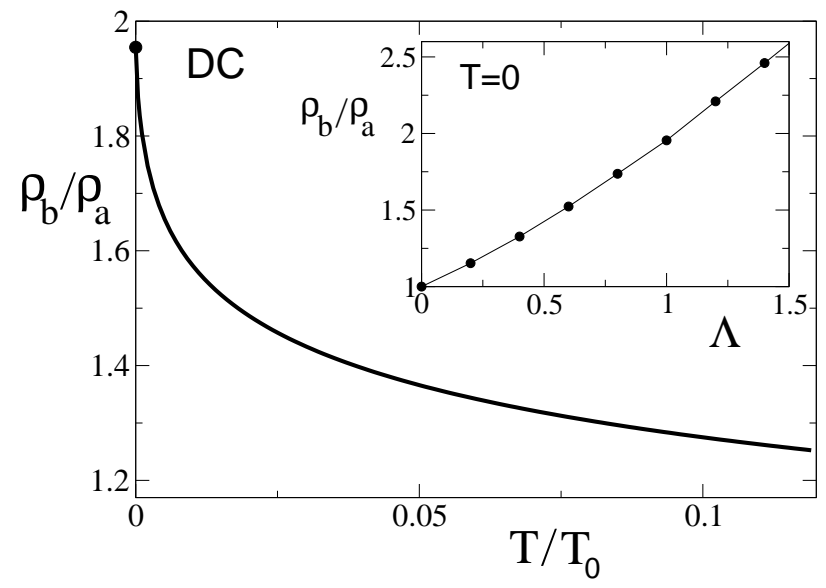

FIG. 3: In-plane DC resistivity anisotropy in the VRH regime, for $\Lambda=1$. Inset: Maximum $(T=0)$ anisotropy versus $\Lambda$.

based on VRH conduction ceases to be valid, as the conduction mechanism changes to impurity band conduction and eventually quasi-metallic behavior. In the most relevant low-temperature range below $T_{V R H}$ (corresponding to largest anisotropy), both the calculated magnitude of $\rho_{b} / \rho_{a}$ and its temperature dependence are very close to the experimental results, $\frac{15}{15}$ although in these experiments the temperature is not low enough to penetrate the "deep" VRH regime $T / T_{0} \lesssim 0.05$ where the anisotropy should increase even further. It should be also noted that there exists quite a bit of uncertainty in the determination of $T_{0}$ and hence in the determination of the exact value of $\rho_{b} / \rho_{a}$ from Fig. 3 .

\section{B. AC Resistivity Anisotropy}

At finite frequency and temperature the calculation of the AC conductivity is a very complicated problem. However in the "quantum" limit when the frequency $\omega \gg T$, the VRH AC conduction is expected to be dominated by resonant absorption by singly occupied pairs (without involvement of phonons), and is usually relevant in doped semiconductors. ${ }^{24}$ The $\mathrm{VRH}$ length in the $\mathrm{AC}$ regime (neglecting for a moment the angular dependence of the states) is logarithmic ${ }^{24} r_{\omega}=(1 / \kappa) \ln (2|\epsilon| / \omega)$, which is the main difference from the DC case. From now on we denote $\Omega \equiv \omega /(2|\epsilon|)$. The formula for $r_{\omega}$ follows from the fact that upon evaluation of the conductivity, the most effective pairs are the ones that satisfy: ${ }^{24} \omega=2 I(r)$, where $I(r)=I_{0} e^{-\kappa r}$ is the overlap integral, and a typical estimate of the prefactor is $I_{0} \approx|\epsilon|$. The functional dependence of the conductivity in $2 \mathrm{D}$ at low frequency $\ln (1 / \Omega) \gg 1$, in the leading logarithmic approximation, is given by the Mott-Shklovskii-Efros expression: $\sigma(\omega) \sim \omega r_{\omega}^{3}\left[\omega+q^{2} / r_{\omega}\right]$, where the second term takes into account the Coulomb interaction in the resonant pair ${ }^{24}$
The generalization of the Mott-Shklovskii-Efros formula to the anisotropic case is straightforward, as it amounts to taking into account the non-exponential (angle-dependent) part of the wave-function, leading to the replacement (with logarithmic accuracy): $\ln (1 / \Omega) \rightarrow$ $\ln \left(\Phi\left(\kappa r_{\omega}, \varphi\right) / \Omega\right) \equiv L_{\omega, \varphi}$, where $\Phi$ is defined in (7). Taking also into account the expressions for $\epsilon, \kappa(5)$, we obtain for the resistivity anisotropy:

$$
\begin{aligned}
& \frac{\rho_{b}(\omega)}{\rho_{a}(\omega)}=\frac{\int_{0}^{2 \pi} \sin ^{2}(\varphi) \mathcal{F}(\omega, \varphi) d \varphi}{\int_{0}^{2 \pi} \cos ^{2}(\varphi) \mathcal{F}(\omega, \varphi) d \varphi} \\
& \mathcal{F}(\omega, \varphi)=L_{\omega, \varphi}^{2}\left(1+\frac{2 \Omega}{1-\Lambda / 2} L_{\omega, \varphi}\right) .
\end{aligned}
$$

The expression (8) is plotted in Fig. 4. Due to the logarithmic dependence, in the theoretical limit of zero frequency the anisotropy would vanish (albeit very slowly), i.e. $\rho_{b}(\omega=0) / \rho_{a}(\omega=0)=1$. However one must keep in mind that the above expressions are not valid at arbitrary low frequencies, as we must have $\omega>T$. Generally we find that the (maximum) AC anisotropy of 30-40\% is somewhat smaller than the DC anisotropy.

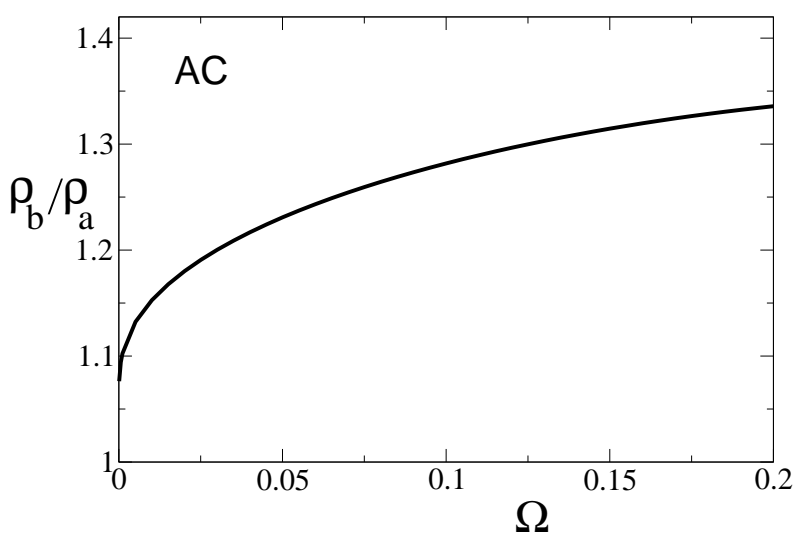

FIG. 4: AC VRH resistivity anisotropy versus $\Omega=\omega /(2|\epsilon|)$ in the regime $T \ll \omega$, and $\ln (1 / \Omega) \gg 1$, for $\Lambda=1$.

When attempting to compare our results to the experimental data of Ref. 16 we realize that $\omega$ and $T$ are not sufficiently low, nor is the difference between them sufficiently high to justify the separation into "quantum" and "thermal" VRH conduction and consequently the Mott-Shklovskii-Efros approach. The temperature used is $T=13 \mathrm{~K}$ and at the lowest frequency of $\omega=20 \mathrm{~cm}^{-1} \approx 2.5 \mathrm{meV}$, estimating for the hole energy $|\epsilon|=\beta \kappa^{2} / 2 \approx 5-10 \mathrm{meV}$, we have $\Omega \sim 0.13-0.25$. Moreover, upon increasing the frequency to $80-100 \mathrm{~cm}^{-1}$, a change of behavior from insulating to conducting takes place, a broad peak develops,$\frac{16}{16}$ and consequently it is not clear to us that the $\mathrm{AC}$ data are ever in a clean $\mathrm{VRH}$ regime. In spite of all this the magnitude of the calculated anisotropy agrees reasonably well with experiment. It should be experimentally possible to lower the temperature (towards the $\mathrm{mK}$ range) as well as $\omega$ in order to 
probe the VRH AC anisotropy more reliably and compare with our theory.

\section{DISCUSSION AND CONCLUSIONS}

We would like to reiterate that in our theory there is no tendency of the holes to form charge stripe-like structures. Nevertheless the resistivity shows anisotropic behavior (and so does the uniform magnetic susceptibility, to be discussed separately). It is particularly important, in our view, that the calculations presented in this work produce quantitative results, and we would thus hope that the successes of the present formulation would stimulate further exploration of the spiral and similar scenarios, not involving any charge-ordering tendencies. The present work is relevant to the spin-glass regime of $\mathrm{La}_{2-x} \mathrm{Sr}_{x} \mathrm{CuO}_{4}, 0.02<x<0.055$. However we have also argued ${ }^{11.12}$ that the spin spiral structure could exist in the superconducting state $x>0.055$. The theory naturally explains why the incommensurate direction, determined from elastic neutron scattering, rotates by $45^{\circ}$ exactly at the insulator-superconductor transition point. On the other hand it is usually argued that at $x=1 / 8$ both in charge-ordered $\mathrm{LNSCO}^{4}$ and $\mathrm{LBCO}_{2}^{25}$ non-collinear spiral order is not consistent with experiment. We would indeed not claim that the spiral ground state is stable at that particular doping since in fact the spiral becomes commensurate with the lattice and therefore significant changes in the ground state could occur due to the spin-lattice coupling.

In conclusion, starting from a spiral ground state which unambiguously follows from the extended $t-J$ model, we have calculated the in-plane anisotropy of the lowtemperature $\mathrm{DC}$ and low-frequency $\mathrm{AC}$ resistivity of $\mathrm{La}_{2-x} \mathrm{Sr}_{x} \mathrm{CuO}_{4}$ in the spin-glass phase. The theory has no fitting parameters and the calculations are performed for the variable-range hopping regime. Within the spiral description the transport anisotropy is due to the anisotropy of the hole wave-function since the hole hops in a non-collinear (spiral) spin background. The AC anisotropy reasonably agrees with experiment in spite of the fact that the data are on the border of the VRH regime. The experimental data for the DC resistivity are well within the VRH regime and here both the calculated magnitude and temperature dependence of the anisotropy agree with experiment very well.

\section{Acknowledgments}

We are grateful to L. Benfatto, J. Haase, and D. Poilblanc for valuable discussions and comments. V.N.K. acknowledges the support of the Swiss National Fund.
* Electronic address: valeri.kotov@epfl.ch

$\dagger$ Electronic address: sushkov@phys.unsw.edu.au

1 K. Orenstein and A. J. Millis, Science 288, 468 (2000).

2 S. Sachdev, Rev. Mod. Phys. 75, 913 (2003).

3 S. A. Kivelson, I. P. Bindloss, E. Fradkin, V. Oganesyan, J. M. Tranquada, A. Kapitulnik, and C. Howald, Rev. Mod. Phys. 75, 1201 (2003).

4 J. M. Tranquada, J. D. Axe, N. Ichikawa, Y. Nakamura, S. Uchida, and B. Nachumi, Phys. Rev. B 54, 7489 (1996).

5 M. Fujita, H. Goka, K. Yamada, J. M. Tranquada, and L. P. Regnault, Phys. Rev. B 70, 104517 (2004).

6 S. Wakimoto, R. J. Birgeneau, M. A. Kastner, Y. S. Lee, R. Erwin, P. M. Gehring, S. H. Lee, M. Fujita, K. Yamada, Y. Endoh, K. Hirota, and G. Shirane, Phys. Rev. B 61, 3699 (2000); M. Matsuda, M. Fujita, K. Yamada, R. J. Birgeneau, M. A. Kastner, H. Hiraka, Y. Endoh, S. Wakimoto, and G. Shirane, Phys. Rev. B 62, 9148 (2000).

7 M. Fujita, K. Yamada, H. Hiraka, P. M. Gehring, S. H. Lee, S. Wakimoto, and G. Shirane, Phys. Rev. B 65, 064505 (2002).

8 S. A. Kivelson, E. Fradkin, and V. J. Emery, Nature 393, 550 (1998).

9 B. I. Shraiman and E. D. Siggia, Phys. Rev. Lett. 62, 1564 (1989); Phys. Rev. B 42, 2485 (1990).

10 O. Zachar, S. A. Kivelson, and V. J. Emery, Phys. Rev. B 57, 1422 (1998).

11 V. N. Kotov and O. P. Sushkov, Phys. Rev. B 70, 195105 (2004), and cited references.

12 O. P. Sushkov and V. N. Kotov, Phys. Rev. Lett. 94, 097005 (2005).
13 N. Hasselmann, A. H. Castro Neto, and C. Morais Smith, Phys. Rev. B 69, 014424 (2004).

14 V. Juricic, L. Benfatto, A. O. Caldeira, and C. Morais Smith, Phys. Rev. Lett. 92, 137202 (2004).

15 Y. Ando, K. Segawa, S. Komiya, and A. N. Lavrov, Phys. Rev. Lett. 88, 137005 (2002).

16 M. Dumm, S. Komiya, Y. Ando, and D. N. Basov, Phys. Rev. Lett. 91, 077004 (2003).

17 W. J. Padilla, M. Dumm, S. Komiya, Y. Ando, and D. N. Basov, cond-mat/0505094

18 P. A. Marchetti, G. Orso, Z. B. Su, and L. Yu, Phys. Rev. B 69, 214514 (2004).

19 M. A. Kastner, R. J. Birgeneau, G. Shirane, and Y. Endoh, Rev. Mod. Phys. 70, 897 (1998).

20 B. Keimer, N. Belk, R. J. Birgeneau, A. Cassanho, C. Y. Chen, M. Greven, M. A. Kastner, A. Aharony, Y. Endoh, R. W. Erwin, and G. Shirane, Phys. Rev. B 46, 14034 (1992).

21 E. Lai and R. J. Gooding, Phys. Rev. B 57, 1498 (1998).

22 M. Suzuki, Phys. Rev. B 39, 2312 (1989).

23 N. F. Mott and E. A. Davis, Electronic Processes in NonCrystalline Materials, 2-nd ed. (Clarendon Press, Oxford, 1979).

24 A. L. Efros and B. I. Shklovskii, in Electron-Electron Interactions in Disordered Systems, edited by A. L. Efros and M. Pollak, p. 409 (North-Holland, Amsterdam, 1985).

25 M. Hücker, G. D. Gu, and J. M. Tranquada, cond-mat/0503417 